\title{
A miR-182 variant and risk of hepatocellular carcinoma in a southern Chinese population
}

\author{
Moqin Qiu ${ }^{1,2}$, Yingchun Liu ${ }^{1,3}$, Qiuling Lin ${ }^{1,2}$, Zihan Zhou ${ }^{1,2}$, Yanji Jiang ${ }^{1,2}$, Rongrui Huo ${ }^{1}$, Xiumei Liang ${ }^{1}$, \\ Xiangyuan $\mathrm{Yu}^{4,5}, \mathrm{Ji} \mathrm{CaO}^{1}$, Xianguo Zhou ${ }^{1,3^{*}}$ and Hongping $\mathrm{Yu}^{1,2,3^{*}}$
}

\begin{abstract}
MicroRNAs (miRNAs) play important roles in the regulation of gene expression at the posttranscriptional level and are involved in human carcinogenesis. The aim of the current study was to investigate the associations between miR-182 single nucleotide polymorphisms and HCC risk in a southern Chinese population. In this case-control study of 863 HCC patients and 908 cancer-free controls, we performed genotyping of miR-182 rs4541843 and assessed its association with HCC risk. We found that individuals carrying the AG/AA genotypes of miR-182 rs4541843 were significantly associated with an increased risk of HCC compared with those carrying the GG genotype (adjusted odds ratio $(\mathrm{OR})=1.71,95 \%$ confidence interval $(C I)=1.07-2.76, P=0.026)$. In the stratified analysis, this increased risk was more pronounced in the subgroups of older individuals (adjusted $\mathrm{OR}=1.98,95 \% \mathrm{Cl}=1.04-3.76, P=$ 0.037), males (adjusted $\mathrm{OR}=1.81,95 \% \mathrm{Cl}=1.09-2.99, P=0.021$ ), and never drinkers (adjusted $\mathrm{OR}=1.84,95 \% \mathrm{Cl}$ $=1.03-3.30, P=0.041$ ). Our results suggested that miR-182 polymorphism rs 4541843 may contribute to the susceptibility to HCC. Our findings require validation in further studies with larger sample sizes.
\end{abstract}

Keywords: Hepatocellular carcinoma, miR-182, Polymorphism, Risk, Association

\section{Introduction}

The incidence of liver cancer in China is markedly higher than that in other geographical areas around the world [1]. In 2018, approximately 841,000 people worldwide were diagnosed with liver cancer, and approximately half of these diagnoses were in China [2]. The major histological subtype of liver cancer is hepatocellular carcinoma (HCC), accounting for approximately $75-80 \%$ of all cases [3]. Several key factors may contribute to $\mathrm{HCC}$, such as hepatitis $\mathrm{B} / \mathrm{C}$ virus (HBV/HCV) infection, cigarette smoking, and heavy drinking, but only a small fraction of exposed individuals eventually develop HCC in their lifetimes [4]. Recently, several genome-wide association studies have identified susceptible loci harboring common single nucleotide polymorphisms

\footnotetext{
* Correspondence: zhouxianguo1001@163.com; yhp268@163.com ${ }^{1}$ Guangxi Medical University Cancer Hospital, Nanning 530021, China Full list of author information is available at the end of the article
}

(SNPs) that are relevant to the risk of $\mathrm{HCC}$, suggesting that genetic factors may be responsible for susceptibility to HCC [5-8].

MicroRNAs (miRNAs) are a class of small non-coding RNAs that can regulate gene expression by pairing with the 3' untranslated region (3' UTR) of mRNAs [9]. To date, more than 1800 precursor miRNAs have been found in the human genome, and aberrant expression of miRNAs has been related to the etiology, diagnosis, and prognosis of many human cancers including HCC [10-12]. MiR-182, located in a 5-kb region of human chromosome 7q32.2, is a member of the miR-183/96/182 cluster [13]. Recently, miR-182 has emerged as a high-priority miRNA in HCC and is involved in the development of HCC by regulating various biological processes, including cell growth, differentiation, migration, invasion, and apoptosis [14-16]. MiR-182 is frequently expressed at high levels in

(c) The Author(s). 2020 Open Access This article is licensed under a Creative Commons Attribution 4.0 International License, which permits use, sharing, adaptation, distribution and reproduction in any medium or format, as long as you give appropriate credit to the original author(s) and the source, provide a link to the Creative Commons licence, and indicate if changes were made. The images or other third party material in this article are included in the article's Creative Commons licence, unless indicated otherwise in a credit line to the material. If material is not included in the article's Creative Commons licence and your intended use is not permitted by statutory regulation or exceeds the permitted use, you will need to obtain permission directly from the copyright holder. To view a copy of this licence, visit http://creativecommons.org/licenses/by/4.0/ The Creative Commons Public Domain Dedication waiver (http://creativecommons.org/publicdomain/zero/1.0/) applies to the data made available in this article, unless otherwise stated in a credit line to the data. 
$\mathrm{HCC}$, and its high expression is correlated with the metastasis, recurrence, and poor prognosis of $\operatorname{HCC}[17,18]$.

Emerging studies have shown that SNPs in miRNAs may alter mRNA expression of their own or target genes, thus leading to tumorigenesis [19-21]. To our knowledge, there are no studies assessing the associations between miR-182 SNPs and the risk of HCC. Given the important roles of $m i R-182$ in tumorigenesis, it is important to determine whether miR-182 SNPs contribute to the susceptibility to HCC. In the current study, we conducted genotyping of $m i R-182$ polymorphisms and assessed their associations with HCC risk in a southern Chinese population.

\section{Materials and methods Study population}

The study population consisted of $863 \mathrm{HCC}$ patients who had undergone surgery between August 2007 and November 2011 in the First Affiliated Hospital of Guangxi Medical University, Affiliated Tumor Hospital of Guangxi Medical University, and First Affiliated Hospital of Guilin Medical University. The tumors were histopathologically confirmed independently as HCC by two pathologists as routine diagnosis. Patients with a prior history of other cancers, metastasized cancers, or previous radiotherapy or chemotherapy before recruitment were excluded. The 908 cancer-free controls were recruited at the same period in Southern China and were genetically unrelated and frequency matched to the cases by age ( \pm 5 years) and sex. Participants with a history of cancer were excluded. The research protocol was approved by the Ethical Committee Review Board of Guangxi Medical University and Guilin Medical University.

With a signed written informed consent form during the interview, each subject enrolled in the study was interviewed to gather the information-related demographic data, history of tobacco and alcohol consumption, and chronic HBV infection through face-to-face interviews conducted by trained investigators. Ever smokers were defined as subjects who had smoked more than 100 cigarettes in their lifetimes; ever drinkers were defined as subjects who had used alcohol at least once a week for more than 1 year. HBV infection was defined as positive for HBV surface antigen (HBsAg). Of the $863 \mathrm{HCC}$ patients, $370 \mathrm{HCC}$ patients had long-term follow-up data for survival analysis. Clinical information (including AFP level, BCLC stage, cancer embolus, and cirrhosis) was collected from medical records. At 3-month intervals, follow-up information on deaths was updated by a trained clinical specialist via telephone. The survival time was calculated in months from the date of tumor resection to the date of death or last follow-up. The patients were followed up until 24 February 2020. A 5-ml peripheral blood sample was obtained from each study subject, of which $1 \mathrm{ml}$ was used to determine the HBV infection status.

\section{SNPs selection and genotyping}

The selected SNPs were screened and identified by NCBI dbSNP database (http://www.ncbi.nlm.nih.gov/) and Haploreg v4.1 (https://pubs.broadinstitute.org/mam mals/haploreg/haploreg.php) in accordance with three criteria: (1) location at the $m i R-182$ gene region; (2) minor allele frequency (MAF) in Chinese Han population > 0.05; and (3) low linkage disequilibrium using an $r^{2}$ threshold of $<0.8$ for each. Ultimately, only one SNP (rs4541843 in miR-182) was selected in this study. Genomic DNA was extracted from peripheral blood by phenol-chloroform extraction and stored at $-80{ }^{\circ} \mathrm{C}$. The selected SNP was genotyped using the Agena MassARRAY genotyping system (Agena, San Diego, CA) according to the manufacturer's instructions. To ensure quality control, $10 \%$ of the samples were randomly selected to repeat the genotyping assays, and the reproducibility was $100 \%$.

\section{Statistical analysis}

Differences in the distributions of demographic characteristics, selected variables and frequencies of genotypes of $m i R-182$ rs4541843 between the cases and controls were evaluated using the $\chi^{2}$ test. Deviation of genotype frequencies in controls was tested with goodness-of-fit $x^{2}$ tests for Hardy-Weinberg equilibrium (HWE). We estimated the associations between $m i R-182$ rs4541843 genotypes and HCC risk by computing the odds ratios (ORs) and their 95\% confidence intervals (CIs) with an unconditional multivariate logistic regression model. The associations between rs4541843 genotypes and HCC risk were also stratified by age, sex, smoking, drinking, and HBV infection. Hazard ratios (HRs) and 95\% CIs were estimated by multivariate Cox proportional hazards model. We performed expression quantitative trait loci (eQTL) analyses with the genomic data from the genotype-tissue expression (GTEx) project to identify correlations between miR-182 rs4541843 and mRNA expression levels of miR182's target genes. Two-sided tests of statistical significance were conducted using SPSS software, version 18.0 (SPSS Institute, Chicago, IL), and the result was considered significant when $P<0.05$ by two-sided tests.

\section{Results}

\section{Characteristics of the study population}

The details of 863 HCC cases and 908 controls enrolled in this study are shown in Table 1 . Because of frequency matching by design, there were no significant differences in the distributions of age or sex between the cases and controls $(P=0.687$ and 0.053 , respectively). However, cases were more likely to be smokers $(43.0 \%$ vs. $25.2 \%, P$ $<0.001)$ and drinkers (38.5 vs. $21.7, P<0.001)$ than the 
Table 1 Frequency distributions of selected variables in HCC cases and controls

\begin{tabular}{|c|c|c|c|}
\hline Variables & Cases $n(\%)$ & Controls $n(\%)$ & $P^{a}$ \\
\hline All subjects & $863(100)$ & $908(100)$ & \\
\hline Age & & & 0.687 \\
\hline$\leq 49$ & $467(54.1)$ & $500(55.1)$ & \\
\hline$>49$ & $396(45.9)$ & 408(44.9) & \\
\hline Sex & & & 0.053 \\
\hline Females & 100(11.6) & $80(8.8)$ & \\
\hline Males & 763(88.4) & $828(91.2)$ & \\
\hline Smoking & & & $<0.001$ \\
\hline Never & $492(57.0)$ & 679(74.8) & \\
\hline Ever & $371(43.0)$ & $229(25.2)$ & \\
\hline Drinking & & & $<0.001$ \\
\hline Never & $531(61.5)$ & $711(78.3)$ & \\
\hline Ever & $332(38.5)$ & 197(21.7) & \\
\hline HBV infection & & & $<0.001$ \\
\hline$(-)$ & 138(16.0) & $821(90.4)$ & \\
\hline$(+)$ & $725(84.0)$ & $87(9.6)$ & \\
\hline
\end{tabular}

${ }^{a}$ Two sides chi-square test

controls. In addition, the percentage of subjects that responded positively to $\mathrm{HBV}$ infection was significantly higher among the HCC cases than among the controls (84.0\% vs. $9.6 \%, P<0.001)$. Therefore, these variables were further adjusted in later multivariate logistic regression analyses for any residual confounding effect.

Associations of miR-182 rs4541843 with the risk of HCC The observed genotype frequencies for this SNP among the control subjects were in agreement with HWE $(P=0.625$ for $m i R-182$ rs4541843). After adjusted for age, gender, smoking status, drinking status, and HBV infection status, we found that the AG/AA genotypes for rs4541843 were significantly associated with an increased risk of HCC compared with the GG genotype (AG/AA vs. GG: adjusted OR $=1.71,95 \% \mathrm{CI}=1.07-2.76, P=0.026)$ (Table 2 ).

To further identify the relationship between $m i R-182$ rs4541843 and HCC risk, the dominant genetic model of

Table 2 Genotype frequencies of rs4541843 in cases and controls and their associations with HCC risk

\begin{tabular}{clllll}
\hline Variants & Cases & Controls & $\boldsymbol{P}^{\mathbf{a}}$ & Adjust OR (95\% Cl) & $\boldsymbol{P}^{\mathbf{b}}$ \\
\hline rs4541843 & & & & & \\
GG & 771 & 833 & 0.222 & 1 & \\
AG & 91 & 74 & & $\mathbf{1 . 7 1 ( 1 . 0 6 - 2 . 7 4 )}$ & $\mathbf{0 . 0 2 7}$ \\
AA & 1 & 1 & & $1.81(0.02-139.1)$ & 0.789 \\
AG/AA & 92 & 75 & 0.084 & $\mathbf{1 . 7 1 ( 1 . 0 7 - 2 . 7 6 )}$ & $\mathbf{0 . 0 2 6}$
\end{tabular}

${ }^{\mathrm{a}}$ Two sides chi-square test

${ }^{b}$ Adjusted for age, sex, smoking, drinking, and HBV infection in logistic regression models this SNP was stratified by subgroups of age, sex, smoking, drinking, and HBV infection. As shown in Table 3, we found that the effect of miR-182 rs4541843 on HCC risk was more pronounced in the older group (adjusted $\mathrm{OR}=1.98,95 \% \mathrm{CI}=1.04-3.76, P=0.037)$, males (adjusted OR $=1.81,95 \% \mathrm{CI}=1.09-2.99, P=0.021$ ), and never-drinking group (adjusted OR $=1.84,95 \% \mathrm{CI}=$ 1.03-3.30, $P=0.041$ ), while no significant interaction between miR-182 rs4541843 genotypes and environmental factors was observed $(P>0.05)$.

\section{Association between miR-182 rs4541843 and prognosis of patients with $\mathrm{HCC}$}

We investigated the associations between SNP rs4541843 and the clinical features of $370 \mathrm{HCC}$ patients, such as AFP level, BCLC stage, cancer embolus, and cirrhosis, but failed to find any significant associations (Table 1S). We performed a multivariate cox regression analysis on the survival of HCC patients, and the result showed that the survival of HCC patients was related to BCLC stage and cancer embolus, but not rs4541843 (Table 2S).

\section{Correlation between rs4541843 genotypes and mRNA expression levels of miR-182' s target genes}

Previous studies have confirmed that $m i R-182$ could promote $\mathrm{HCC}$ progression by targeting multiple genes, such as MTSS1, TP53INP1, and RASA1 [18, 22, 23]. So, we conducted the eQTL analysis to evaluate the correlations between SNP rs4541843 genotypes and the expression levels of these genes using the data of the GTEx

Table 3 Stratified analysis between rs4541843 polymorphism and HCC risk

\begin{tabular}{|c|c|c|c|c|}
\hline \multirow[t]{2}{*}{ variables } & \multicolumn{2}{|c|}{ rs4541843 (cases/controls) } & \multirow{2}{*}{$\begin{array}{l}\text { Adjust OR }(95 \% \mathrm{Cl}) \\
\text { AG/AA vs. GG }\end{array}$} & \multirow[t]{2}{*}{$P^{a}$} \\
\hline & GG & AG/AA & & \\
\hline \multicolumn{5}{|l|}{ Age } \\
\hline$\leq 49$ & $418 / 455$ & $49 / 45$ & $1.44(0.71-2.91)$ & 0.310 \\
\hline$>49$ & $353 / 378$ & $43 / 30$ & $1.98(1.04-3.76)$ & 0.037 \\
\hline \multicolumn{5}{|l|}{ Sex } \\
\hline Females & $90 / 72$ & $10 / 8$ & $1.19(0.30-4.63)$ & 0.807 \\
\hline Males & $681 / 761$ & $82 / 67$ & $1.81(1.09-2.99)$ & 0.021 \\
\hline \multicolumn{5}{|l|}{ Smoking } \\
\hline Never & $432 / 624$ & $60 / 55$ & $1.79(0.99-3.24)$ & 0.055 \\
\hline Ever & $339 / 209$ & $32 / 20$ & $1.62(0.73-3.60)$ & 0.235 \\
\hline \multicolumn{5}{|l|}{ Drinking } \\
\hline Never & $468 / 655$ & $63 / 56$ & $1.84(1.03-3.30)$ & 0.041 \\
\hline Ever & $303 / 178$ & 29/19 & $1.51(0.67-3.40)$ & 0.326 \\
\hline \multicolumn{5}{|c|}{ HBV infection } \\
\hline$(-)$ & $119 / 752$ & 19/69 & $1.73(0.98-3.05)$ & 0.060 \\
\hline$(+)$ & $652 / 81$ & $73 / 6$ & $1.62(0.68-3.86)$ & 0.277 \\
\hline
\end{tabular}

${ }^{a}$ Adjusted for age, sex, smoking, drinking, and HBV infection in logistic regression models 
database. Although we found that the A allele of SNP rs4541843 appeared to be correlated with the decreased expression levels of MTSS1, TP53INP1, and RASA1 in the liver tissues, the correlations between them did not reach statistically significant level (Supplementary Figure 1).

\section{Discussion}

In the present study, we investigated whether miR-182 polymorphisms were associated with the risk of HCC in a southern Chinese population, and found that miR-182 rs4541843 AG/AA genotypes were significantly associated with an evidently increased HCC risk, especially in older group, males and never-drinking group. Our findings suggest, for the first time, that genetic variant of miR-182 might contribute to the development of HCC in this population.

$M i R-182$ has been reported to act as a tumor promoter in HCC, and its upregulation has been shown to be associated with the development, metastasis, recurrence, and poor prognosis of HCC. For instance, aberrant miR-182 expression can promote $\mathrm{HCC}$ metastasis by targeting metastasis suppressor 1 (MTSS1), and miR-182 may be used as a diagnostic marker or potential therapeutic target in HCC [18]. Cao et al. investigated the function of $m i R-182-5 p$ and found that the over-expression of miR-182-5p was related to poor prognosis and early recurrence in HCC. Subsequent functional experiments showed that miR-182-5p could repress FOXO3a expression by directly targeting its 3'UTR, activating the AKT/ FOXO3a pathway to promote HCC progression [22]. Du et al. found that miR-182 was induced in HCC cells under hypoxia and promoted angiogenesis by targeting RASA1 [24]. Additionally, miR-182 plays an important role in drug resistance and upregulated miR-182induced cisplatin resistance in HCC cells by regulating tumor protein 53-induced nuclearprotein 1 (TP53INP1) [23]. So, we conducted the eQTL analysis to evaluate the correlations between SNP rs4541843 genotypes and the mRNA expression levels of miR-182's target genes using the data of the GTEx database. Although we found that the A allele of the SNP rs4541843 appeared to be correlated with the decreased expression levels of MTSS1, TP53INP1, and RASA1 in the liver tissues, the correlations between them did not reach statistically significant level. Tumorigenesis is a complex process, it is reasonable to speculate that miR-182 rs4541843 might simultaneously alter the expression of multiple target genes, and subsequently contribute to the risk of HCC. However, further functional studies and association studies with larger sample sizes are warranted to confirm our findings.

Several studies have reported that single nucleotide polymorphisms in miRNA genes may influence individual susceptibility to cancers including HCC [25-27]. Xu et al. investigated the influence of miR-146a rs2910164
( $\mathrm{G}>\mathrm{C}$ ) on individual susceptibility to HCC and found that males with the GG genotype of miR-146a gene were 2-fold more susceptible to HCC than those carrying the $\mathrm{CC}$ genotype $(\mathrm{OR}=2.02,95 \% \mathrm{CI}=1.06-3.85, P=$ 0.034) [28]. Further investigation revealed that the GG genotype conferred a higher expression level of mature miR-146a, therefore was more susceptible to carcinogens. Liu et al. showed that the A-to-G base change in rs999885 in the promoter region of the miR-106b-25 cluster may lead to an increased risk for HCC in HBVpersistent carriers by altering the expression of the miR106b-25 cluster [29]. Bei et al. showed that SNP of rs1135519 could modulate miR-122 expression and contribute to the genetic susceptibility of HCC, either independently or together with rs9966765 in miR-122 [30]. In our study, we selected one common SNP located in miR-182 and found that the miR-182 rs4541843 AG/AA genotypes were associated with a significantly increased risk of HCC. In the stratification analysis by variables, we observed that this significant association with the risk of HCC was particularly pronounced in the older group, males and the never-drinking group. However, studies of larger sample sizes among different populations are needed to confirm our findings.

Some potential limitations of our study should be considered. First, because our study was a hospital-based case-control study with an unavoidable selection bias, we applied a rigorous design in selecting study subjects, and controls were well matched to cases according to age and sex to minimize potential biases. Second, although the sample size of our study was large, a relatively small size in some subgroup analyses may have limited the statistical power. Finally, the precise molecular mechanism underlying the observed effects require further investigation.

In conclusion, our study provides evidence supporting the notion that the miR-182 polymorphism might be associated with HCC risk in a southern Chinese population. Further studies with larger sample sizes and functional studies are warranted to validate our findings.

\section{Supplementary information}

Supplementary information accompanies this paper at https://doi.org/10. 1186/s40246-020-00289-X.

\footnotetext{
Additional file 1: Table 1S. The associations between miR-182 rs4541843 polymorphism and clinical features of HCC patients. Table 2 S. Cox regression analysis of the prognosis of HCC Patients.

Additional file 2: Figure S1. The correlation between miR-182 rs4541843 and mRNA expression of its target genes in the liver tissues from the genotype-tissue expression database.
} 


\section{Authors' contributions}

Mogin Qiu and Yingchun Liu contributed equally to this work. QMQ and LYC performed the research and wrote the paper. LQL, ZZH, and JYJ collected blood samples and extracted genomic DNA, LXM, CJ, and XYY were in charge of epidemiological investigation, HRR analyzed the data, and ZXG and YHP participated in the conception and design. All authors read and approved the final manuscript

\section{Funding}

This study was supported by the National Natural Science Foundation of China (grant nos. 81660567 and 81460516), Natural Science Foundation of Guangxi Province of China (grant nos. 2018GXNSFDA050012 and 2015GXNSFCB139007), The Key Research and Development Project of Guangxi (grant nos. AB18050020 and AA18221001), and International Communication of Guangxi Medical University Graduate Education.

\section{Availability of data and materials}

The data used to support the findings of this study are available from the corresponding author upon request.

\section{Ethics approval and consent to participate}

Not applicable.

\section{Consent for publication}

Not applicable.

\section{Competing interests}

The authors declare that they have no competing interests.

\section{Author details}

${ }^{1}$ Guangxi Medical University Cancer Hospital, Nanning 530021, China. ${ }^{2}$ School of Public Health, Guangxi Medical University, Nanning 530021, China. ${ }^{3}$ Guangxi Cancer Molecular Medical Engineering Research Center, Nanning 530021, China. ${ }^{4}$ Affiliated Hospital of Guilin Medical University, Guilin 541004, China. ${ }^{5}$ School of Public Health, Guilin Medical University, Guilin 541004, China.

\section{Received: 28 April 2020 Accepted: 6 October 2020}

Published online: 15 October 2020

\section{References}

1. Torre LA, Bray F, Siegel RL, Ferlay J, Lortet-Tieulent J, Jemal A. Global cancer statistics, 2012. CA Cancer J Clin. 2015:65:87-108.

2. Bray F, Ferlay J, Soerjomataram I, Siegel RL, Torre LA, Jemal A. Global cancer statistics 2018: GLOBOCAN estimates of incidence and mortality worldwide for 36 cancers in 185 countries. CA Cancer J Clin. 2018;68:394-424.

3. Villanueva A. Hepatocellular Carcinoma. N Engl J Med. 2019:380:1450-62

4. Lafaro KJ, Demirjian AN, Pawlik TM. Epidemiology of hepatocellular carcinoma. Surg Oncol Clin N Am. 2015;24:1-17.

5. Li Y, Zhai Y, Song Q, Zhang H, Cao P, Ping J, et al. Genome-Wide Association Study Identifies a New Locus at 7q21.13 Associated with Hepatitis B Virus-Related Hepatocellular Carcinoma. Clin Cancer Res. 2018;24: 906-15.

6. Zhang H, Zhai Y, Hu Z, Wu C, Qian J, Jia W, et al. Genome-wide association study identifies 1 p36.22 as a new susceptibility locus for hepatocellular carcinoma in chronic hepatitis B virus carriers. Nat Genet. 2010;42:755-8.

7. Matsuura K, Sawai H, Ikeo K, Ogawa S, lio E, Isogawa M, et al. Genome-Wide Association Study Identifies TLL1 Variant Associated With Development of Hepatocellular Carcinoma After Eradication of Hepatitis C Virus Infection. Gastroenterology. 2017;152:1383-94.

8. Lee MH, Huang YH, Chen HY, Khor SS, Chang YH, Lin YJ, et al. Human leukocyte antigen variants and risk of hepatocellular carcinoma modified by hepatitis C virus genotypes: A genome-wide association study. Hepatology. 2018;67:651-61.

9. Ambros V. The functions of animal microRNAs. Nature. 2004:431:350-5.

10. Bartel DP. MicroRNAs: genomics, biogenesis, mechanism, and function. Cell. 2004;116:281-97.

11. Xu J, Zhu X, Wu L, Yang R, Yang Z, Wang Q, et al. MicroRNA-122 suppresses cell proliferation and induces cell apoptosis in hepatocellular carcinoma by directly targeting Wnt/beta-catenin pathway. Liver Int. 2012;32:752-60.
12. Ura S, Honda M, Yamashita T, Ueda T, Takatori H, Nishino R, et al. Differentia microRNA expression between hepatitis $B$ and hepatitis $C$ leading disease progression to hepatocellular carcinoma. Hepatology. 2009;49:1098-112.

13. Woldemichael BT, Jawaid A, Kremer EA, Gaur N, Krol J, Marchais A, et al. The microRNA cluster miR-183/96/182 contributes to long-term memory in a protein phosphatase 1-dependent manner. Nature Communications. 2016;7:12594.

14. Li P, Sheng C, Huang L, Zhang H, Huang L, Cheng Z, et al. MiR-183/-96/-182 cluster is up-regulated in most breast cancers and increases cell proliferation and migration. Breast Cancer Res. 2014;16:473.

15. Segura MF, Hanniford D, Menendez S, Reavie L, Zou X, Alvarez-Diaz S, et al. Aberrant miR-182 expression promotes melanoma metastasis by repressing FOXO3 and microphthalmia-associated transcription factor. Proc Natl Acad Sci U S A. 2009;106:1814-9.

16. Wang X, Li H, Cui L, Feng J, Fan Q. MicroRNA-182 suppresses clear cell renal cell carcinoma migration and invasion by targeting IGF1R. Neoplasma. 2016; 63:717-25.

17. Chen L, Chu F, Cao Y, Shao J, Wang F. Serum miR-182 and miR-331-3p as diagnostic and prognostic markers in patients with hepatocellular carcinoma. Tumour Biol. 2015;36:7439-47.

18. Wang J, Li J, Shen J, Wang C, Yang L, Zhang X. MicroRNA-182 downregulates metastasis suppressor 1 and contributes to metastasis of hepatocellular carcinoma. BMC cancer. 2012;12:227.

19. Shi TY, Chen XJ, Zhu ML, Wang MY, He J, Yu KD, et al. A pri-miR-218 variant and risk of cervical carcinoma in Chinese women. BMC Cancer. 2013;13:19.

20. Xu Y, Liu L, Liu J, Zhang Y, Zhu J, Chen J, et al. A potentially functional polymorphism in the promoter region of $\mathrm{miR}-34 \mathrm{~b} / \mathrm{c}$ is associated with an increased risk for primary hepatocellular carcinoma. Int J Cancer. 2011;128:412-7

21. Xie K, Liu J, Zhu L, Liu Y, Pan Y, Wen J, et al. A potentially functional polymorphism in the promoter region of let-7 family is associated with survival of hepatocellular carcinoma. Cancer Epidemiology. 2013;37:998-1002.

22. Cao MQ, You AB, Zhu XD, Zhang W, Zhang YY, Zhang SZ, et al. miR-182-5p promotes hepatocellular carcinoma progression by repressing FOXO3a. J Hematol Oncol. 2018:11:12

23. Du C, Weng X, Hu W, Lv Z, Xiao H, Ding C, et al. Hypoxia-inducible MiR-182 promotes angiogenesis by targeting RASA1 in hepatocellular carcinoma. J Exp Clin Cancer Res. 2015;34:67

24. Qin J, Luo M, Qian H, Chen W. Upregulated miR-182 increases drug resistance in cisplatin-treated HCC cell by regulating TP53INP1. Gene. 2014; 538:342-7.

25. Wang Q, Yu X, Li Q, Qin L, Tan S, Zeng X, et al. Association between miR199a rs74723057 and MET rs1621 polymorphisms and the risk of hepatocellular carcinoma. Oncotarget. 2016;7:79365-71.

26. An J, Liu J, Liu L, Liu Y, Pan Y, Huang $M$, et al. A genetic variant in primary miR-378 is associated with risk and prognosis of hepatocellular carcinoma in a Chinese population. PLoS One. 2014;9:e93707.

27. Chu YH, Hsieh MJ, Chiou HL, Liou YS, Yang CC, Yang SF, et al. MicroRNA gene polymorphisms and environmental factors increase patient susceptibility to hepatocellular carcinoma. PLoS One. 2014;9:e89930.

28. Xu T, Zhu Y, Wei QK, Yuan Y, Zhou F, Ge YY, et al. A functional polymorphism in the miR-146a gene is associated with the risk for hepatocellular carcinoma. Carcinogenesis. 2008;29:2126-31.

29. Liu Y, Zhang Y, Wen J, Liu L, Zhai X, Liu J, et al. A genetic variant in the promoter region of miR-106b-25 cluster and risk of HBV infection and hepatocellular carcinoma. PLoS One. 2012;7:e32230.

30. Bei C, Liu S, Yu X, Qiu M, Tang B, Liao W, et al. Single Nucleotide Polymorphisms in miR-122 Are Associated with the Risk of Hepatocellular Carcinoma in a Southern Chinese Population. Biomed Res Int. 2018;2018: 1540201.

\section{Publisher's Note}

Springer Nature remains neutral with regard to jurisdictional claims in published maps and institutional affiliations. 